\title{
Optimization of convex functions with fenchel biconjugation and duality
}

\author{
Vinod Kumar Bhardwaj* \\ Department of Mathematics, G.L.A. University, Mathura, India
}

(C2018 ACCENTS

\begin{abstract}
Analysis of conjugation operations to induce a bijection between proper closed convex functions and to discuss the problems of boundedness of closed convex proper functions using continuity of conjugates. Present study shows a great contribution of biconjugation of convex functions in optimization. Fenchel biconjugation describes the relation of duality in optimization. Problems of finite dimensional Lagrangian convex duality theory and problems on duality gap are comparative of primal and dual solutions in convex optimization.
\end{abstract}

\section{Keywords}

Convex functions, Fenchel biconjugation, Lagrangian duality, Optimization.

\section{Introduction}

The assumption of a closed function is a dissimilar to previous definition by Rockafellar et al. [1, 2], although they coincide for proper functions but our assumption easily extends to infinite dimensions [3]. Von Neumann's minimax theorem had both the sets $\mathrm{C}$ and D simplices [4]. Brouwer proved this by fixed point theorem. Fisher introduced information function useful in signal reconstruction [5]. Interior point methods depend on the inequality in Logarithmic homogeneity [6]. No one can consider biconjugate unless it itself has a closed convex epigraph, since any conjugate function must have a closed convex epigraph. Nonempty compact convex sets in $\mathrm{E}$ and everywhere-finite sub linear functions have Fenchel conjugacy between them [7].

Finite dimensional Lagrangian convex duality theorem provides comparative study on problems on duality gap and zero duality gap and analysis of dual and primal solutions in convex optimization. Elegant feature of theory is the duality between a convex function and its Fenchel conjugate. The study of optimization is significantly depends on its control to describe duality theory for convex programming problems. The perfectly well-defined dual problems without any assumptions on the functions and g show the 'weak duality inequality' [8]. This is great feature of finite-dimensional convex duality theory and its development in infinite dimensions [9].

*Author for correspondence
A duality gap can be easily defined by a problem where the primal value is strictly greater than the dual value [10]. We investigate conditions ensuring that there is no duality gap and the result highlights the proof of the Lagrangian necessary conditions which in fact describes the existence of a dual optimal solution $[11,12]$. To prove that there is no duality gap we consider following two different approaches: first uses the Slater condition to force attainment in the dual problem and second (dual) approach uses compactness to force attainment in the primal problem [2]. The problem is said to be normal when the value function $\mathrm{v}$ is lower semi continuous at 0 [1] and the problem is said to be stable if $\partial v(\mathrm{O}) \neq \phi(\operatorname{or} v(\mathrm{O})=-\infty)[13]$. On the gap functions of prevariational inequalities Yang [11] and on the dual gap function for variational inequalities Zhang et al. [12] are some good reference work on duality gap functions. Chong et.al derived Sufficient and necessary conditions for the stable Fenchel duality and for the total Fenchel duality, also proved some sufficient and necessary conditions for the strong Fenchel duality and the strong converse Fenchel duality using the properties of the epigraph of the conjugated functions [14]. Jeyakumar et al. developed a robust theorem of the alternative for parameterized convex inequality systems using conjugate analysis and derives a new robust characteristic cone constraint qualification necessary and sufficient for strong duality between the robust equivalent and its Lagrangian dual [15]. Wang et.al establishes some total and strong Fenchel dualities for convex optimization problems accompanying data uncertainty within the framework of robust 
optimization in locally convex Hausdorff vector spaces [16]. Fajardo used generalized convex conjugation theory instead in place of Fenchel conjugation and construct an alternative dual problem, using the perturbational approach, for a general optimization one defined on a separated locally convex topological space [17].

In the paper following points are focused:

- Biconjugates of proper convex functions are developed to discuss the optimality conditions.

- Relationship between convex function and its biconjugate and the contribution of these in optimization is described.

- Lagrangian Duality theorem is used to describe the duality gap and zero duality gap between convex function and its conjugate.

\section{Preliminaries and definitions}

We have seen that for many important convex functions $h: E \rightarrow(-\infty,+\infty]$, the biconjugate $h^{* *}$ agrees identically with $\mathrm{h}$. The bipolar cone theorem shows $\delta_{K}=\delta_{K}^{* *}$ for any closed convex cone $\mathrm{K}$. In the paper we isolate exactly the circumstances when $\mathrm{h}=h^{* *}$. We can easily check that $h^{* *}$ is a minorant of $\mathrm{h}$ (that is, $\boldsymbol{h}^{* *} \leq \boldsymbol{h}$ point wise). Our explicit plan in the paper is to find conditions on a point $\mathrm{x}$ in $\mathbf{E}$ guaranteeing $h^{* *}(x)=h(x)$. This becomes the key relationship for the study of duality in optimization. As we see in the paper, the conditions we need are both geometric and topological. This is neither particularly astonishing nor rigid. It is very to consider a function with its biconjugate without having a closed convex epigraph, since any conjugate function must have a closed convex epigraph. On the other side, this restriction is not mainly strong, as the previous study shows that convex functions fundamentally have strong continuity properties.

Consider the function $\boldsymbol{h}: \boldsymbol{E} \rightarrow[-\infty,+\infty]$ is closed if its epigraph is a closed set. Which indicates that $\mathrm{h}$ is lower semi continuous at a point $\mathrm{x}$ in $\mathbf{E}$ if

$$
\liminf h\left(x^{r}\right)\left(=\lim _{x \rightarrow \infty} \inf _{r \geq s} h\left(x^{r}\right)\right) \geq h(x)
$$

for any sequence $x^{r} \rightarrow x$. A function $h: E \rightarrow[-\infty,+\infty]$ is lower semi continuous if it is lower semi continuous at every point in $\mathbf{E}$ : this is in fact corresponding to $\mathrm{h}$ being closed, the result holds if and only if $\mathrm{h}$ has closed level sets. Any two functions $\mathrm{h}$ and $\mathrm{g}$ satisfying $h \leq g$ (in which case we call $\mathrm{h}$ a minorant of g) must satisfy $h^{*} \geq g^{*}$, and hence $h^{* *} \leq g^{* *}$.

\section{Fenchel biconjugation}

Theorem 3.1 (Fenchel biconjugation) The properties below are equivalent, for any function $h: E \rightarrow[-\infty,+\infty]:$

(a) $\mathrm{h}$ is closed and convex;

(b) $\mathrm{h}=h^{* *}$;

(c) for all points $\mathrm{x}$ in $\mathbf{E}$,

$h(x)=\sup \{\alpha(x) \mid \alpha$ an affine min orant of $h\}$

Hence the conjugacy operation induces a bijection between proper closed convex functions.

Proof. We can assume $h$ is proper. Since conjugate functions are always closed and convex we know property (b) implies property (a). Also, any affine minorant $\alpha$ of h satisfies $\alpha=\alpha^{* *} \leq h^{* *} \leq \boldsymbol{h}$, and hence property (c) implies (b). It remains to show (a) implies (c).

Fix a point $x^{\circ}$ in E. Assume first $x^{\circ} \in \operatorname{cl}(\operatorname{dom} h)$, and fix any real $\mathrm{r}<\mathrm{h}\left(x^{\circ}\right)$. Since $h$ is closed, the set $\{x \mid h(x)>r\}$ is open, so there is an open convex neighborhood $\mathrm{U}$ of $x^{\circ}$ with $\mathrm{h}(\mathrm{x})>\mathrm{r}$ on $\mathrm{U}$. Now note that the set dom $h$ กcont $\delta_{U}$ is nonempty, so we can apply the Fenchel theorem to deduce that some element $\phi$ of $\mathbf{E}$ satisfies

$r \leq \inf _{x}\left\{h(x)+\delta_{U}(x)\right\}=\left\{-h^{*}(\phi)-\delta_{U}^{*}(\phi)\right\}$

Now define an affine function $\alpha(\cdot)=\langle\phi, \cdot\rangle+\delta_{U}^{*}(\phi)+r$. Inequality (3.2) shows that $\alpha$ minorizes $\mathrm{h}$, and by definition we know $\alpha\left(x^{\circ}\right) \geq r$. Since $r$ was arbitrary, (c) follows at the point $\mathrm{x}=x^{\circ}$.

Suppose on the other hand $x^{\circ}$ does not lie in cl (dom $\mathrm{h})$. By the Basic separation theorem there is a real b and a nonzero element a of $\mathbf{E}$ satisfying $\left\langle a, x^{\circ}\right\rangle>\mathrm{b} \geq\langle\mathrm{a}, \mathrm{x}\rangle$, for all points $\mathrm{x}$ in dom $\mathrm{h}$. 
The argument in the preceding paragraph shows there is an affine minorant $\alpha$ of h. But now the affine function $\alpha(\cdot)+k(\langle a, \cdot\rangle-b)$ is a minorant of $\mathrm{h}$ for all $k=1,2, \ldots \ldots$. Evaluating these functions at $\mathrm{x}=x^{\circ}$ proves property (c) at $x^{\circ}$. The final remark follows easily.

We can immediately deduce that a closed convex function $\quad h: E \rightarrow[-\infty,+\infty]$ equals its biconjugate if and only if it is proper or identically $+\infty$ or $-\infty$. Restricting the conjugacy bijection to finite sub linear functions gives the following result.

Corollary 3.2 (Support functions) Fenchel conjugacy induces a bijection between everywherefinite sub linear functions and nonempty compact convex sets in $\mathbf{E}$ :

(a) If the set $C \subset \boldsymbol{E}$ is compact, convex and nonempty then the support function $\delta_{C}^{*}$ is everywhere finite and sub linear.

(b) If the function $h: E \rightarrow R$ is sub linear then $h^{*}=\delta_{C}$, where the set

$C=\{\phi \in E \mid\langle\phi, d\rangle \leq h(d)$ for all $d \in E\}$

is nonempty, compact and convex.

Proof. See in previous section. Conjugacy offers a convenient way to recognize when a convex function has bounded level sets.

Theorem 3.3 (Moreau-Rockafellar) A closed convex proper function on $\mathbf{E}$ has bounded level sets if and only if its conjugate is continuous at 0 .

Proof. By Proposition, a convex function $\boldsymbol{h}: \boldsymbol{E} \rightarrow(-\infty,+\infty]$ has bounded level sets if and only if it satisfies the growth condition

$$
\liminf _{\|x\| \rightarrow \infty} \frac{f(x)}{\|x\|}>0
$$

Since $f$ is closed we can check that this is equivalent to the existence of a minorant of the form $\varepsilon\|\cdot\|+k \leq f(\cdot)$, for some constants $\varepsilon>0$ and $\mathrm{k}$.

Taking conjugates, this is in turn equivalent to $f^{*}$ being bounded above near 0 , and the result then follows by Theorem (Local boundedness).
Strict convexity is also easy to recognize via conjugacy, using the following results.

Theorem 3.4 (Strict-smooth duality) A proper closed convex function on $\mathbf{E}$ is essentially strictly convex if and only if its conjugate is essentially smooth.

What can we say about $h^{* *}$ when the function $h: E \rightarrow[-\infty,+\infty]$ is not necessarily closed? To answer this question we introduce the idea of the closure of $\mathrm{h}$, denoted $\mathrm{cl} \mathrm{h}$, defined by

$\operatorname{epi}(\mathrm{cl} h)=\operatorname{cl}($ epi $h)$

It is easy to verify that $\mathrm{cl} \mathrm{h}$ is then well-defined. The definition immediately implies $\mathrm{cl} \mathrm{h}$ is the largest closed function minorizing $\mathrm{h}$. Clearly if $\mathrm{h}$ is convex, so is $\mathrm{cl} \mathrm{h}$.

Proposition 3.5 (Lower semi continuity and closure) A convex function

$h: E \rightarrow[-\infty,+\infty]$ is lower semi continuous at a point $\mathrm{x}$ where it is finite if and only if $f(\mathrm{x})=(\mathrm{cl} f$ (x). In this case $f$ is proper.

We can now answer the question we posed at the beginning of the section.

Theorem 3.6 Suppose the function $\boldsymbol{h}: \boldsymbol{E} \rightarrow[-\infty,+\infty]$ is convex.

(a) If $h^{* *}$ is somewhere finite then $h^{* *}=\mathrm{cl} \mathrm{h}$.

(b) For any point $\mathrm{x}$ where $\mathrm{h}$ is finite, $\mathrm{h}(\mathrm{x})=h^{* *}(x)$ if and only if $\mathrm{h}$ is lower semi continuous at $\mathrm{x}$.

Proof. Observe first that since $h^{* *}$ is closed and minorizes h, we know $h^{* *} \leq \mathrm{cl} h \leq \boldsymbol{h}$. If $\boldsymbol{h}^{* *}$ is somewhere finite then $h^{* *}$ (and hence $\mathrm{cl} \mathrm{h}$ ) is never $-\infty$, by applying Proposition 3.6 (Lower semi continuity and closure) to $h^{* *}$. On the other hand, if $\mathrm{h}$ is finite and lower semi continuous at $\mathrm{x}$ then Proposition 3.6 shows $\mathrm{cl} \mathrm{h}(\mathrm{x})$ is finite, and applying the proposition again to $\mathrm{cl} \mathrm{h}$ shows once more that $\mathrm{cl}$ $h$ is never $-\infty$. In either case, the Fenchel biconjugation theorem implies $c l h=(c l h)^{* *} \leq h^{* *} \leq c l h, \quad$ so cl $h=h^{* *}$.

Part (a) is now immediate, while part (b) follows by using Proposition 3.6 once more. 


\section{Lagrangian duality}

Consider a convex program like that of studied in section-3.2

$\left\{\begin{array}{cc}\text { inf } & f(x) \\ \text { subject to } & g(x) \leq \mathrm{O} \\ & x \in E\end{array}\right.$

Here, the function $f$ and the components $g_{1}, g_{2}, \ldots, g_{m}: E \rightarrow(-\infty,+\infty]$ are convex, and satisfy $\phi \neq \operatorname{dom} f \bigcap_{1}^{m} \operatorname{dom} g_{i}$. As before, the Lagrangian function $\boldsymbol{L}: E \times \boldsymbol{R}_{+}^{m} \rightarrow(-\infty,+\infty]$ is defined by $L(x ; \lambda)=f(x)+\lambda^{T} g(x)$.

Notice that the Lagrangian encapsulates all the information of the primal problem (4.3.1): clearly

$$
\sup _{\lambda \in R_{+}^{m}} L(x ; \lambda)=\left\{\begin{array}{l}
f(x), \text { if } x \text { is feasible } \\
+\infty, \text { otherwise }
\end{array}\right.
$$

so if we denote the optimal value of (4.3.1) by $p \in[-\infty,+\infty]$, we could rewrite the problem in the following form:

$$
p=\inf _{x \in E} \sup _{\lambda \in R_{+}^{m}} L(x ; \lambda)
$$

This makes it rather natural to consider an associated problem:

$$
d=\sup _{\lambda \in R_{+}^{m}} \inf _{x \in E} L(x ; \lambda)
$$

Where $d \in[-\infty,+\infty]$ is called the dual value. Thus the dual problem consists of maximizing over vectors $\lambda$ in $\boldsymbol{R}_{+}^{m}$ the dual function $\Phi(\lambda)=\inf _{x} L(x ; \lambda)$.

This dual problem is perfectly well-defined without any assumptions on the functions $f$ and $g$. It is an easy exercise to show the 'weak duality inequality' $p \geq d$. Notice $\Phi$ is concave.

It can happen that the primal value $\mathrm{p}$ is strictly larger than the dual value $d$. In this case we say there is a duality gap. In this section we investigate conditions ensuring there is no duality gap. As the chief tool in our analysis is the primal value function $v: R^{m} \rightarrow[-\infty,+\infty]$, defined by

$$
v(b)=\inf \{f(x) \mid g(x) \leq b\} \text {. }
$$

Below we summarize the relationships between these various ideas and pieces of notation.
Proposition 4.1 (Dual optimal value)

(a) The primal optimal value $\mathrm{p}$ is $v(\mathrm{O})$.

(b) The conjugate of the value function satisfies

$v^{*}(-\lambda)=\left\{\begin{array}{l}-\Phi(\lambda), \quad \text { if } \lambda \geq 0 \\ +\infty, \quad \text { otherwise }\end{array}\right.$

(c) The dual optimal value $\mathrm{d}$ is $v^{* *}(\mathrm{O})$.

Proof. Part (a) is just the definition of p. Part (b) follows from the identities

$$
\begin{aligned}
v^{*}(-\lambda) & =\sup \left\{-\lambda^{T} b-v(b) \mid b \in R^{m}\right\} \\
& =\sup \left\{-\lambda^{T} b-f(x) \mid g(x)+z=b, x \in \operatorname{dom} f, b \in R^{m}, z \in R_{+}^{m}\right\} \\
& =\sup \left\{-\lambda^{T}(g(x)+z)-f(x) \mid x \in \operatorname{dom} f, z \in R_{+}^{m}\right\} \\
& =-\inf \left\{f(x)+\lambda^{T} g(x) \mid x \in \operatorname{dom} f\right\}+\sup \left\{-\lambda^{T} z \mid z \in R_{+}^{m}\right\} \\
& =\left\{\begin{array}{l}
-\Phi(\lambda), \text { if } \lambda \geq 0 \\
+\infty, \quad \text { otherwise }
\end{array}\right.
\end{aligned}
$$

Finally, we observe

$d=\sup _{\lambda \in R_{+}^{m}} \Phi(\lambda)=-\inf _{\lambda \in R_{+}^{m}}-\Phi(\lambda)=-\inf _{\lambda \in R_{+}^{m}} v^{*}(-\lambda)=v^{* *}$

So part (c) follows. Notice the above result does not use convexity.

The reason for our interest in the relationship between a convex function and its biconjugate should now be clear, in light of parts (a) and (c) above.

Corollary 4.2 (Zero duality gap) Suppose the value of the primal problem (4.1) is finite. Then the primal and dual values are equal if and only if the value function $v$ is lower semi continuous at 0 . In this case the set of optimal dual solutions is $-\partial v(0)$.

Proof. By the previous result, there is no duality gap exactly when the value function satisfies $v(\mathrm{O})=v^{* *}(\mathrm{O})$, so Theorem 3.8 proves the first assertion.

By part (b) of the previous result, dual optimal solutions $\lambda$ are characterized by the property $\mathbf{O} \in \partial \boldsymbol{v}^{*}(-\lambda), \quad$ or $\quad$ equivalently, $v^{*}(-\lambda)+v^{* *}(\mathrm{O})=\mathbf{O}$. But we know $v(0)=v^{* *}(0)$, so this property is equivalent to the condition $-\lambda \in \partial v(\mathrm{O})$. 
The above result gave a new on the proof of the Lagrangian necessary conditions: this actually demonstrates the existence of a dual optimal solution. To prove that there is no duality gap we consider following two different approaches: first uses the Slater condition to force attainment in the dual problem and second (dual) approach uses compactness to force attainment in the primal problem.

Theorem 4.3 (Dual attainment) If the Slater condition holds for the primal problem (4.1) then the primal and dual values are equal, and the dual value is attained if finite.

Proof. If $\mathrm{p}$ is $-\infty$ there is nothing to prove, since we know $p \geq \boldsymbol{d}$. If on the other hand $\mathrm{p}$ is finite then, as in the proof of the Lagrangian necessary conditions, the Slater condition forces $\partial v(0) \neq \phi$. Hence $v$ is finite and lower semi continuous at 0 and the result follows by Corollary 4.3.6 (Zero duality gap). An indirect way of stating the Slater condition is that there is a point $\hat{x}$ in $\mathbf{E}$ for which the set $\left\{\lambda \in R_{+}^{m} \mid L(\hat{x} ; \lambda) \geq \alpha\right\}$ is compact for all real $\alpha$.

The second approach uses a 'dual' condition to ensure the value function is closed.

Theorem 4.4 (Primal attainment) Suppose that the functions

$f, g_{1}, g_{2}, \ldots, g_{m}: E \rightarrow(-\infty,+\infty] \quad$ are closed, and that for some real $\hat{\lambda} \geq 0$ and some vector $\hat{\lambda}$ in $R_{+}^{m}$, the function $\hat{\lambda}_{\mathrm{o}} f+\hat{\lambda}^{T} g$ has compact level sets. Then the value function $\mathrm{v}$ defined by equation (4.3.4) is closed, and the infimum in this equation is attained when finite. Consequently, if the functions $f, g_{1}, g_{2}, \ldots, g_{m}$ are in addition convex and the dual value for the problem (4.1) is not $-\infty$, then the primal and dual values, $\mathrm{p}$ and $\mathrm{d}$, are equal, and the primal value is attained when finite.

Proof. If the points $\left(b^{r}, s_{r}\right)$ lie in epi $v$ for $r=1,2, \ldots \ldots \ldots$, and approach the point $(\mathrm{b} ; \mathrm{s})$, then for each integer $\mathrm{r}$ there is a point $x^{r}$ in $\mathbf{E}$ satisfying $f\left(x^{r}\right)=s_{r}+r^{-1}$ and $g\left(x^{r}\right) \leq b^{r}$ Hence we deduce $\left(\hat{\lambda}_{0} f+\hat{\lambda}^{T} g\right)\left(x^{r}\right) \leq \hat{\lambda}_{0}\left(s_{r}+r^{-1}\right)+\hat{\lambda}^{T} b^{r} \rightarrow \hat{\lambda}_{0} s+\hat{\lambda}^{T} b$.
By the compact level set assumption, the sequence ( $x^{r}$ ) has a subsequence converging to some point $\bar{x}$, and since all the functions are closed, we know $f(\bar{x}) \leq s$ and $g(\bar{x}) \leq b$. We deduce $v(b) \leq s$, so (b; s) lies in epi $v$ as we required. When $v(b)$ is finite, the same argument with $\left(b^{r}, s_{r}\right)$ replaced by $(b, v(b))$ for each $r$ shows the infimum is attained.

If the functions $f, g_{1}, g_{2}, \ldots, g_{m}$ are convex then we know $v$ is convex. If $\mathrm{d}$ is $+\infty$ then, then again from the inequality $p \geq \boldsymbol{d}$, there is nothing to prove. If $d\left(=v^{* *}(\mathrm{O})\right)$ is finite then Theorem3.8 shows $v^{* *}=c l v$, and the above argument shows cl $v=v$. Hence $p=v(\mathrm{O})=v^{* *}(\mathrm{O})=\boldsymbol{d}$, and the result follows.

\section{Conclusion}

Any proper convex function $h$ with an affine minorant has its biconjugate $h^{* *}$ somewhere finite. (In fact, because $\mathbf{E}$ is finite-dimensional, $h^{* *}$ is somewhere finite if and only if $h$ is proper) Notice that if either the objective function $f$ or any one of the constraint functions $g_{1}, g_{2}, \ldots, g_{m}$ has compact level sets then the compact level set condition in the primal attainment results hold. Relationship between convex function and its biconjugate results a great contribution in optimization. Lagrangian Duality theorem describe duality gap and zero duality gap between convex function and its biconjugate.

\section{Acknowledgment}

None.

\section{Conflicts of interest}

The author has no conflicts of interest to declare.

\section{References}

[1] Rockafellar RT. Convex analysis. Princeton University Press Princeton. New Jersey. 1997.

[2] Rockafellar RT, Wets RJ. Variational analysis. Springer Science \& Business Media; 2009.

[3] Ekeland I, Temam R. Convex analysis and variational problems. SIAM; 1999.

[4] Dem'yanov VF, Malozemov VN. Introduction to minimax. Courier Corporation; 1990.

[5] Borwein JM, Lewis AS, Noll D. Maximum entropy spectral analysis using first order information. part I: 
fisher information and convex duality. Mathematics of Operations Research. 1996; 21:442-68.

[6] Nesterov Y, Nemirovskii A. Interior-point polynomial algorithms in convex programming. SIAM; 1994.

[7] Phelps RR. Convex functions, monotone operators and differentiability. Lecture Notes in Mathematics. 1989; 1364:425-30.

[8] Peressini AL, Sullivan FE, Uhl JJ. The mathematics of nonlinear programming. New York: Springer-Verlag; 1988.

[9] Holmes RB. Geometric functional analysis and its applications. Springer Science \& Business Media; 1975.

[10] Wright SJ. Primal-dual interior-point methods. SIAM; 1997.

[11] Yang XQ. On the gap functions of prevariational inequalities. Journal of Optimization Theory and Applications. 2003; 116(2):437-52.

[12] Zhang J, Wan C, Xiu N. The dual gap function for variational inequalities. Applied Mathematics and Optimization. 2003; 48(2):129-48.

[13] Bertsekas DP. Nonlinear programming. Belmont: Athena Scientific; 1999.
[14] Li C, Fang D, López G, López MA. Stable and total fenchel duality for convex optimization problems in locally convex spaces. SIAM Journal on Optimization. 2009; 20(2):1032-51.

[15] Jeyakumar V, Li GY. Strong duality in robust convex programming: complete characterizations. SIAM Journal on Optimization. 2010; 20(6):3384-407.

[16] Wang M, Fang D, Chen Z. Strong and total Fenchel dualities for robust convex optimization problems. Journal of Inequalities and Applications. 2015; 2015:70.

[17] Fajardo MD, Vidal J. Stable strong Fenchel and Lagrange duality for evenly convex optimization problems. Optimization. 2016; 65(9):1675-91.

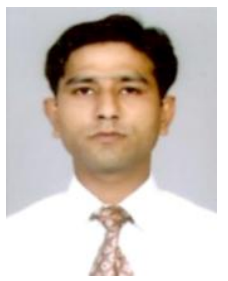

Vinod Kumar Bhardwaj received his Ph.D. degree in Mathematics from the Dr. B. R. Ambedkar University, Agra (India). He is currently Assistant Professor at the Department of Mathematics of GLA University, Mathura (India). His research interests are optimization problems.

Email: dr.vinodbhardwaj@gmail.com 